\title{
The effect of individualized, theory-based counselling intervention on active aging and quality of life among older people (the AGNES intervention study)
}

\author{
Taina Rantanen ${ }^{1}$ (1) Mary Hassandra ${ }^{1} \cdot$ Katja Pynnönen $^{1} \cdot$ Sini Siltanen $^{1} \cdot$ Katja Kokko $^{1} \cdot$ Laura Karavirta $^{1}$. \\ Markku Kauppinen $^{1} \cdot$ Sarianna Sipilä ${ }^{1} \cdot$ Milla Saajanaho $^{1} \cdot$ Erja Portegijs $^{1}$
}

Received: 10 January 2020 / Accepted: 12 March 2020 / Published online: 1 April 2020

(c) The Author(s) 2020

\begin{abstract}
Background We define active aging as a striving for activities as per one's goals, capacities and opportunities.

Aim To test the 1-year counselling intervention effects on active aging.

Methods In this two-arm single-blinded randomized controlled trial, the intervention group received individually tailored counselling supporting autonomous motivation for active life (one face-to-face session, four phone calls and supportive written material, $n=101)$ and the control group written health information $(n=103)$. Participants were community-dwelling men and women aged 75 or 80 years with intermediate mobility function and without cognitive impairment. The primary outcome was active aging total score measured with the University of Jyväskylä Active Aging Scale (UJACAS, range 0-272, higher values indicate more activity) and secondary outcomes were its subscores for goals, ability, opportunity and activity (range 0-68) and a quality of life (QoL) score. Measures took place at pre-trial, mid-trial (6 months) and post-trial (12 months), except for QoL only pre and post-trial. Data were analyzed with intention-to-treat principles using GEE-models.

Results The UJACAS total score increased in the intervention group slightly more than in the control group (group by time $p$-value $=0.050$, effect size 0.011 , net benefit $2 \%$ ), but the group effect was not statistically significant. A small effect was observed for the activity subscore $(p=0.007)$.

Discussion The individualized counselling supporting autonomous motivation for active life increased the UJACAS score slightly.

Conclusions It may be possible to promote active aging with individualized counselling, but the effect is small and it is unclear whether the change is meaningful.
\end{abstract}

Keywords Behavior change $\cdot$ Aging $\cdot$ Quality of life $\cdot$ Mobility $\cdot$ Randomized controlled trial

\section{Introduction}

Activity refers to doing things and relates to all essential fields of human life. Many older people state that avoiding passiveness and sustaining activeness underpins positive life experience [1, 2]. With increasing age, however, people may give up important activities e.g. due to declining functional capacity [3,4], which may concur with lower quality of life [4].

Taina Rantanen

taina.rantanen@jyu.fi

1 Faculty of Sport and Health Sciences, Gerontology Research Center, University of Jyväskylä, Jyväskylä, Finland
Defining and assessing an active approach to life during aging has mostly concerned physical activity and paid or unpaid work [5]. However, adopting a broader view on activity by emphasizing participation in any meaningful activities based on individual predispositions may provide a more inclusive picture of active aging [6]. Older people have many personal goals related to diverse activities [3]. As people often act in line with their goals [3], most likely their activities are also highly diverse. We acknowledge this diversity in our recent definition of active aging as "the striving for activities relating to a person's goals, functional capacities and opportunities" [7] which we aim to capture in our 17-item University of Jyväskylä Active Aging Scale (UJACAS). We developed the scale in a multi-phase process, and the total score represents the unidimensional latent 
construct of active aging. The items assess active agency in essential life areas outlined in the International Classification of Functioning, Disability and Health [8]. The four subscores assess what people want to do, what they are able to do, whether they have the opportunity to do the activity, and how much or how often they do the activity [7]. The novel aspects of UJACAS are that it captures diverse forms of activity in old age, includes generic activities that are in principle possible for all, and does not provide strict objective criteria for performing the activity. For example, for one person 'helping others' may mean taking part in formal and regular volunteer work, while for somebody else it may mean watering the neighbor's plants while they are travelling.

We observed in our pilot data that the higher the active aging score was, the better were the indicators of quality of life [7] suggesting that increased activity in any essential life areas may enhance positive life experiences. Quality of life is multidimensional and refers to perceptions of one's position in life relative to one's goals and living environment [9]. Quality of life is increasingly included as an outcome in health interventions [10]. Due to the wide scope of the concept, many different interventions may improve quality of life among older people [10,11].

As active aging takes diverse forms, we concluded that individualized counselling intervention could be a feasible way to promote it. Counselling is an interaction process that helps the participant to see things more clearly and possibly from a different point of view. In this study, the idea was to increase awareness of meaningful and desirable activities that are likely to yield personal benefits, to set new selfselected activity goals, promote autonomous motivation and lend support to positive changes in activity [12]. We decided to use a counselling approach that integrates two socio-cognitive theoretical models, the self-determination theory [13] and the theory of planned behavior [14]. The self-determination theory concerns motivation as our intrinsic predisposition to behave in positive ways, and it explains the motivation underlying everyday activities in older people [15-17]. The theory of planned behavior links one's beliefs and behavior and explains how older individuals intend to behave [18-22]. A recent meta-analysis [23] suggested that integrating these two theories may provide a solid basis for planning a behavior change intervention. The integrated model has been used to elucidate the behavior of older adults [24], but earlier studies on counselling interventions based on the integrated model do not exist.

The aim of this 12-month randomized controlled trial was to investigate the effects of the individualized counselling intervention on the UJACAS total score (primary outcome). In addition, we examined the effects of the intervention on the four UJACAS sub-scores and quality of life (secondary outcomes). The hypothesis was that the UJACAS total score will increase $10 \%$ in intervention group and no change or slight decrease will be observed in the control group. For future hypothesis building, we conducted preplanned subgroup analyses for the UJACAS total score.

\section{Methods}

The study protocol has been published earlier [12]. The study design was a single-blinded randomized controlled trial with two research arms. The parallel groups are the "Counselling group", serving as the intervention group, and a "Health information group", serving as the control group. Trained research assistants collecting data at all time-points were unaware of the group allocation.

\section{Participants}

We recruited participants from the AGNES cohort study $[25,26]$, which is an observational study on active aging of people aged 75,80 , or 85 years living independently in the municipality of Jyväskylä, Finland. Participants for the cohort study were recruited based on random samples drawn from the Population Information System administered by the Population Register Center based on their place of residence and year of birth. All who consented and were able to communicate with researchers were included in the cohort study. Inclusion criteria for the current RCT were age 75 or 80 years, willingness to participate, a baseline score between 52.3 and 90.0 on the University of Alabama at Birmingham Life-Space Assessment (LSA) [27, 28], and a score of 25 or higher on the cognitive function test Mini Mental State Examination (MMSE) [29]. Life-space mobility describes the spatial area where a person moves on a regular basis, the frequency of moving and the assistance needed [26]. Consequently, it describes a person's prospects to take part in different life-situations. People with life-space mobility composite score in the recruitment range represent the "middle group" in the population, excluding those with mobility restrictions and those with most extensive mobility behavior. We selected the MMSE score 25 or higher as an inclusion criterion because counselling is a cognitive intervention and requires the participant to process the topics discussed. We excluded people who took part in other ongoing intervention studies. Recruitment started in October 2017 and continued until end of August in 2018. Measurements were completed in August 2019.

\section{Randomization}

We used stratified randomization for age and sex with a 1:1 allocation to ensure a balance of participant characteristics in each group. The randomization sequence was created 
using Stata 15.0 statistical software (StataCorp, College Station, TX) by the study statistician, who immediately after generation of the random allocation sequence sealed them in envelopes. For each person, the counsellor opened the envelope after completion of all baseline data collection.

\section{Measurements}

Participants were assessed pre-trial, mid-trial at 6 months, and post-trial at 12 months by trained research assistants. All assessments were conducted face-to-face in the participants' homes by trained interviewers using computer assisted personal interviewing. In addition, prior to randomization the counsellor conducted a brief interview over the phone for profiling purposes.

\section{Intervention}

The active aging counselling intervention has been described earlier [12]. Briefly, the counselling group participated in a 90-min, face-to-face, individual counselling session on the premises of the University of Jyväskylä following a semistructured protocol of questions. After the initial face-to-face session, participants received four phone counselling sessions, at 1, 3, 6 and 9 months after the face-to-face counselling session to provide them with additional support, feedback and encouragement. Participants received four printed newsletters listing activities organized for older people in our town, and the success stories of people who are living an active life. During their face-to-face counselling session, participants received an information booklet on active aging from the counsellor. The booklet is 64 pages long and provides information about goal setting for active life, and activities and behaviors that promote active aging.

The central elements of the counselling intervention were to support the autonomous motivation for and foresee the benefits of increased or new activities according to the goals set by the participant. We also provided information about different local activities for older people. The 17 activities composing the UJACAS were used as the starting point for the goal setting discussions. As a result, the targets were several different active behaviors that differed between individuals. Depending of the preferences of the participant, we encouraged any self-selected activities that take place outside the home or that involve social interaction, such as attending events, going for walks with a friend or joining a club. Our unpublished pilot data analyses suggested that such activities have largest 'spill-over' effects on other activities and quality of life. We followed the guidelines of the Medical Research Council (MRC) framework for complex interventions [30] and implementation research [31-33].

To support autonomous motivation for self-selected activities, individuals set their goals, planned their actions and monitored their progress in change process. The individualization of the counselling sessions rested on pre-existing data on health, social contacts, mood, loneliness and preferred activities and goals.

The health information group received printed general health information material similar to what is used in health care services for older people. We posted the printed materials (brochures, booklets etc.) to the health information group participants during months $1,3,6$, and 9 . The themes were exercise, nutrition, cardiovascular diseases, and type II diabetes, respectively.

\section{Main outcome}

The main outcome measure is the total score of the University of Jyväskylä Active Aging Scale (UJACAS) [7]. The UJACAS consists of 17 items: practicing memory, using a computer, advancing matters in one's own life, exercising, enjoying the outdoors, taking care of one's appearance, crafting or DIY, making the home cozy and pleasant, helping others, maintaining friendships, getting to know new people, balancing personal finances, making one's days interesting, practicing artistic hobbies, participating in events, advancing societal/communal matters, and doing things according to one's world view. For each item, participants were asked to evaluate on a Likert scale their striving to accomplish the activity, their ability and opportunity to perform the activity and their amount or frequency of doing the activity during the 4 weeks immediately prior to the measurement. Response options ranged from zero (not at all/very low) to four (very much/very high) with verbalization of rating depending on the wording of the question. Subscores (range 0-68) for the striving, ability, opportunities and activity were then calculated and the composite score (range 0-272) were calculated with higher scores reflecting greater striving, better ability or opportunities, and higher activity. Similarly, a higher composite score (range 0-272) indicates a higher level of active aging. We have previously shown that the scale has good psychometric properties, test-retest reliability and that it assesses a unidimensional latent construct of active aging [7].

\section{Secondary outcomes}

The secondary outcomes include the active aging subscores and quality of life score.

Quality of life was assessed pre- and post-trial with a short version of the Quality of Life Questionnaire for Older People (OPQOL-brief). The scale includes 13 items related to a person's satisfaction with life overall, health, participation, social relationships and financial situation. Answers are given on a scale from one (strongly disagree) to five 
(strongly agree). The sum score ranges from 13 to 65, with higher scores indicating higher quality of life [34].

\section{Additional baseline measures}

Age and sex were obtained as part of the sampling data drawn from the Population Register Centre in the context of recruitment. Education was assessed by the number of completed years of education [35]. Perceived financial situation was assessed on a scale ranging from one (very good) to five (very poor). Physical health was assessed based on self-reported physician-diagnosed diseases. A co-morbidity index was calculated from a checklist of diseases prompted by ten categories of chronic diseases and an open-ended question about any other physician-diagnosed chronic conditions [36]. Lower-extremity physical performance was assessed by the Short Physical Performance Battery (SPPB) [37]. It comprises tests on standing balance, walking speed over a 3-m distance, and chair-stands. Established cut-off points were used to score each task from zero to four points, higher scores indicating better performance. Participants unable to perform a test were assigned the score zero. A sum score was calculated (range 0-12) when at least two tests were completed. Cognitive functioning was tested with MMSE [29] with higher scores indicating better results.

\section{Statistical analyses}

Power calculations were conducted for the primary outcome, the UJACAS total score assessed at 12 months. A total of 168 participants were needed for a $90 \%$ probability to detect a treatment difference at a two-sided 0.05 significance level, if the true difference in the main outcome between the intervention and the control group is $10 \%$. We assumed that some of the participants are vulnerable, so we decided to recruit 200 participants to allow for the potential attrition rate of $20 \%$ during the 12-month intervention. The estimate of $10 \%$ difference between the groups was based on approximations from two earlier intervention studies [37, 38].

Baseline characteristics were summarized using means and standard deviation, or percentages. Intervention adherence was calculated based on the proportion attending the scheduled face-to-face and phone counselling sessions. We analyzed the effect of the intervention on the primary outcome, the active aging total score, with intention-to-treat approach according to randomized groups. To compare intervention effects linear Generalized Estimation Equations (GEE) for repeated measures were computed using unstructured correlations, where group $\times$ time interaction indicates if change differs between the groups. This method adjusts for potential baseline differences in the outcomes. GEE modeling takes into account all available data when estimating intervention effects. Effect size was calculated from per protocol analyses and describes the net difference in changes between groups. The group difference represents the difference in the level of the active aging score over the entire follow-up. The secondary endpoints were analyzed similarly as the primary outcome.

The trial is registered at ISRCTN_-ISRCTN16172390: promoting well-being through active aging.

\section{Results}

From October 2017 until end of August in 2018, we screened 416 participants of the AGNES-cohort study for the current RCT. Of them, 204 were eligible and were randomized either to the active aging counselling group $(N=101)$ or to the health information group $(N=103)$ (Fig. 1). Baseline characteristics between the two groups were comparable (Table 1). All participants were aged 75 or 80 years and approximately a quarter of both groups were 80 years of age and approximately $60 \%$ were women. The sole statistically significant difference observed was for SPPB score with health information group receiving slightly higher results at the baseline.

\section{Intervention adherence}

Of the 101 participants randomized into the counselling group, two did not receive any part of the intervention while seven participants cancelled their participation later on during the intervention. Altogether, $8.9 \%$ discontinued in the intervention. In the control group, seven participants (6.8\%) discontinued.

\section{Adverse events}

The counselling and health information groups did not differ in incidence of adverse events related to health and social relationships during the intervention study. In the counselling group, $20 \%$ of the participants got a new disease, in $20 \%$ existing disease got worse, $8 \%$ had an accident, $17 \%$ had a medical operation, and $44 \%$ some other health problem. Proportions in the health information group were $18 \%, 22 \%, 5 \%$, $11 \%$ and $39 \%$ respectively. Death of a close person occurred for $11 \%$ in the counselling and $19 \%$ in the health information group. In the counselling group, $26 \%$ worried about health or life situation of a close person and $6 \%$ reported of narrowed friendship network. Respective proportions for the health information group were 28 and $2 \%$.

One person reported negative effects directly related to the study, which was feeling exhausted during the 6-month interview. 
Fig. 1 The flow chart of the study

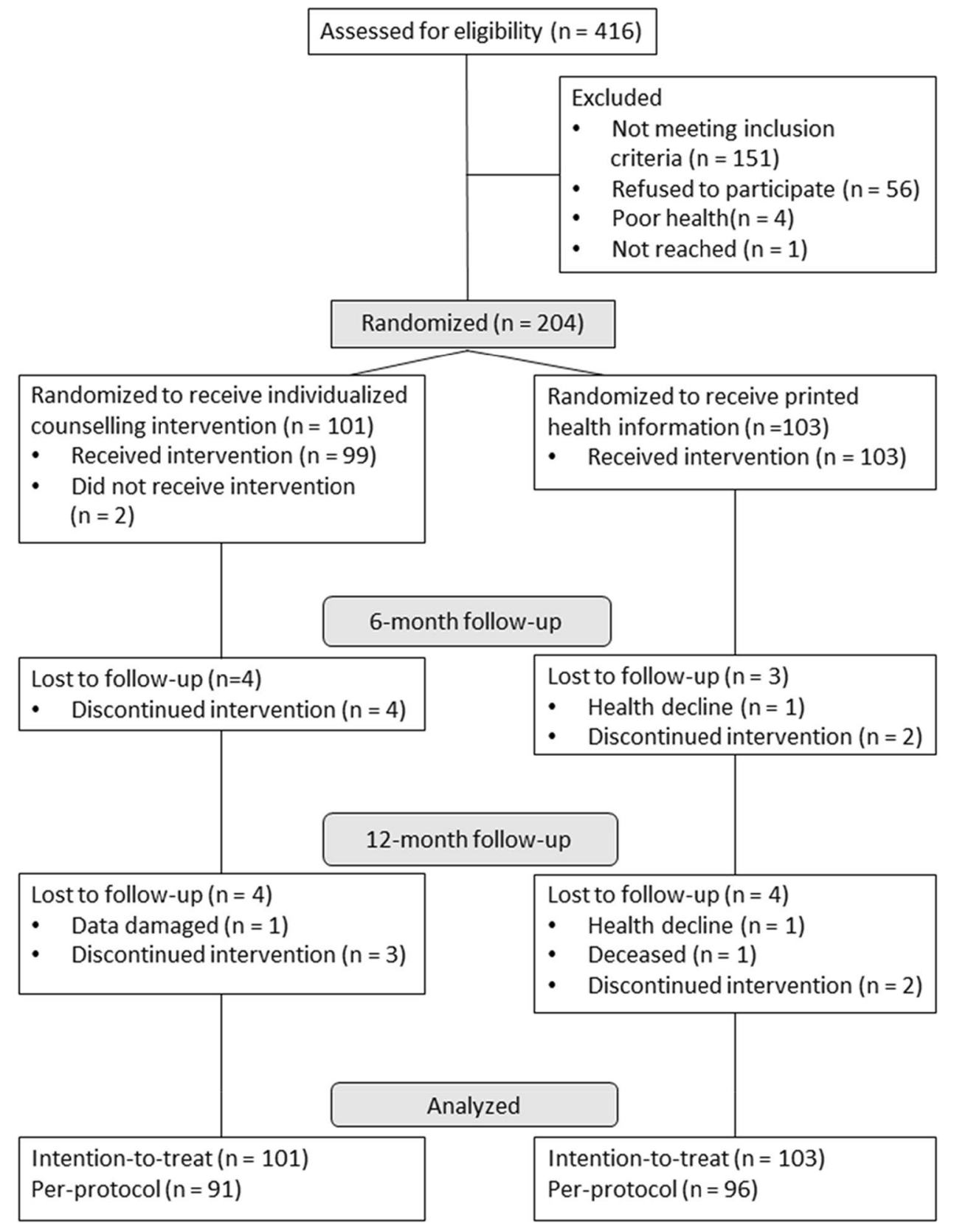

\section{Active aging scores}

According to the principles of the ITT-analyses all participants randomized were included in the GEE models testing the intervention effects. Table 2 shows that the counselling intervention increased the active aging total score compared to the health information (group by time $p$-value $=0.050$ ). The net difference in change was $2 \%$ for the benefit of the intervention.

For the active aging subscores, a significant group by time interaction was observed for the amount of activity $(p=0.007)$ accompanied by a significant group difference $(p=0.045)$ with intervention group getting higher values
(Table 2). For the other subscores, statistically significant effects were not found.

The mean quality of life score at the baseline was 56.2 (SD 4.8) and the intervention had no effect on quality of life, the groups did not differ, and statistically significant change over time was not observed.

\section{Preplanned sub-group analyses}

The preplanned subgroup analyses for the main outcome were conducted for age, sex, socioeconomic position, MMSE and SPPB (Table 3). The intervention increased active aging total score among those with self-rated poor 
Table 1 The baseline characteristics of the participants according to randomized groups

\begin{tabular}{lll}
\hline & Counselling $(n=101)$ & $\begin{array}{l}\text { Health } \\
\text { information } \\
(n=103)\end{array}$ \\
\hline Age, 80 -year-olds, $n(\%)$ & $26(25.7)$ & $26(25.2)$ \\
Women, $n(\%)$ & $61(60.4)$ & $63(61.2)$ \\
Tertiary education, $n(\%)$ & $41(41.0)$ & $53(51.5)$ \\
Education, mean (SD), years & $11.4(3.9)$ & $12.0(3.7)$ \\
Perceived financial situation, very good/good, $n(\%)$ & $60(60)$ & $66(66)$ \\
Life-space mobility, mean (SD), score & $75.5(9.2)$ & $74.7(9.3)$ \\
MMSE, mean (SD), score & $27.8(1.5)$ & $28.2(1.2)^{*}$ \\
CES-D, mean (SD), score & $8.12(7.1)$ & $7.0(6.1)$ \\
SPPB, mean (SD), score & $10.6(1.6)$ & $11.0(1.2)^{*}$ \\
\hline
\end{tabular}

$C E S-D$ Center for Epidemiologic Studies Depression Scale, SPPB Short Physical Performance Battery, MMSE mini-mental state examination, ${ }^{*} p=<0.05$

Table 2 Means and standard deviations (SD) for the active ageing total score (main outcome) and active ageing subscores at the baseline, 6 months and 12 months in the randomized groups

\begin{tabular}{|c|c|c|c|c|c|c|c|}
\hline Outcome & $\begin{array}{l}\text { Baseline } \\
\text { Mean (SD) }\end{array}$ & $\begin{array}{l}6 \text { Months } \\
\text { Mean (SD) }\end{array}$ & $\begin{array}{l}12 \text { Months } \\
\text { Mean (SD) }\end{array}$ & $\begin{array}{l}\text { Group } \\
p \text {-value }\end{array}$ & $\begin{array}{l}\text { Time } \\
p \text {-value }\end{array}$ & $\begin{array}{l}\text { Group by time } \\
p \text {-value }\end{array}$ & $\begin{array}{l}\text { Interaction } \\
\text { effect size }\end{array}$ \\
\hline Active ageing, total score & & & & 0.121 & 0.153 & 0.050 & 0.011 \\
\hline Counselling group & $204.1(26.3)$ & $200.6(25.7)$ & $207.2(24.6)$ & & & & \\
\hline Health information group & $207.9(25.3)$ & $203.0(24.6)$ & $206.6(24.1)$ & & & & \\
\hline Will to act, subscore & & & & 0.483 & 0.614 & 0.369 & 0.005 \\
\hline Counselling group & $45.7(9.3)$ & $45.1(8.6)$ & $46.8(8.7)$ & & & & \\
\hline Health information group & $46.2(8.7)$ & $46.2(8.6)$ & $46.8(8.2)$ & & & & \\
\hline Ability to act, subscore & & & & 0.284 & 0.023 & 0.162 & 0.007 \\
\hline Counselling group & $62.1(6.0)$ & $60.7(6.8)$ & $62.0(6.4)$ & & & & \\
\hline Health information group & $62.4(5.4)$ & $60.9(6.3)$ & $61.3(6.5)$ & & & & \\
\hline Opportunity to act, subscore & & & & 0.231 & 0.065 & 0.279 & 0.003 \\
\hline Counselling group & $54.3(9.2)$ & $52.3(9.5)$ & $54.1(8.8)$ & & & & \\
\hline Health information group & $55.5(7.9)$ & $53.0(8.5)$ & $54.5(7.8)$ & & & & \\
\hline Amount of activity, subscore & & & & 0.045 & 0.793 & 0.007 & 0.019 \\
\hline Counselling group & $42.1(8.4)$ & $42.4(7.5)$ & $44.4(7.1)$ & & & & \\
\hline Health information group & $43.9(8.6)$ & $43.0(7.6)$ & $44.0(7.6)$ & & & & \\
\hline
\end{tabular}

Generalized estimation equations with $p$-values for the main effects of group and time, and group by time interaction shown for each outcome. Effect size is calculated for per protocol participants

or at most satisfactory financial situation (group by time $p$-value 0.033 ) and those with high SPPB score (group by time $p$-value 0.038).

\section{Discussion}

The intervention increased active aging total score statistically significantly, but the effect size and net benefits were small. The results suggest that it may be possible to promote active aging with individualized counselling targeting autonomous motivation for self-selected activities, however, it is unclear whether the observed small changes are meaningful. This is the first study to target a wide range of self-selected activities with individualized counselling supporting autonomous motivation.

We planned the study based on the results of our earlier observational studies and randomized controlled trials. We reported previously that having more personal goals correlates with greater life-space mobility, which exemplifies a person's exposure to out-of-home goings-on [38]. Any activity outside the home will increase physical activity and social interaction and potentially help prevent social isolation and physical inactivity, see e.g. [39, 40]. Our earlier physical activity counselling intervention increased physical activity and maintained better mobility [41, 42]. For the current study, we extended the scope to any self-selected activity that takes place outside home or that is done with 
Table 3 Means and standard deviations (SD) for the active ageing total score for counselling group vs. health information group according to subgroups at the baseline, 6 months and 12 months in the randomized groups

\begin{tabular}{|c|c|c|c|c|c|c|c|}
\hline Subgroup & $N$ & $\begin{array}{l}\text { Baseline } \\
\text { Mean (SD) }\end{array}$ & $\begin{array}{l}6 \text { Months } \\
\text { Mean (SD) }\end{array}$ & $\begin{array}{l}12 \text { Months } \\
\text { Mean (SD) }\end{array}$ & $\begin{array}{l}\text { Group } \\
p \text {-value }\end{array}$ & $\begin{array}{l}\text { Time } \\
p \text {-value }\end{array}$ & $\begin{array}{l}\text { Group by time } \\
p \text {-value }\end{array}$ \\
\hline Women & & & & & 0.065 & 0.865 & 0.123 \\
\hline Counselling group & 61 & $203.8(26.1)$ & $176.7(68.6)$ & $206.8(25.0)$ & & & \\
\hline Health information group & 63 & $210.6(24.3)$ & $193.0(55.2)$ & $209.1(22.8)$ & & & \\
\hline Men & & & & & 0.081 & 0.598 & 0.279 \\
\hline Counselling group & 40 & $204.6(26.9)$ & $200.9(23.6)$ & $207.8(24.4)$ & & & \\
\hline Health information group & 40 & $203.7(26.4)$ & $192.6(40.9)$ & $202.7(25.8)$ & & & \\
\hline 75 year-olds & & & & & 0.309 & 0.068 & 0.093 \\
\hline Counselling group & 75 & $205.4(24.8)$ & $193.1(47.2)$ & $206.3(24.0)$ & & & \\
\hline Health information group & 77 & $208.0(26.0)$ & $193.0(46.3)$ & $205.4(25.2)$ & & & \\
\hline 80 year-olds & & & & & 0.421 & 0.811 & 0.805 \\
\hline Counselling group & 26 & $200.5(30.7)$ & $166.8(74.8)$ & $210.3(27.0)$ & & & \\
\hline Health information group & 26 & $207.5(23.5)$ & $192.1(60.4)$ & 209.9 (20.9) & & & \\
\hline Financial situation very good/good & & & & & 0.863 & 0.299 & 0.460 \\
\hline Counselling group & 60 & $210.8(25.0)$ & $101.1(38.8)$ & $210.9(23.7)$ & & & \\
\hline Health information group & 63 & $208.1(24.8)$ & $192.0(54.0)$ & $208.0(14.5)$ & & & \\
\hline Financial situation satisfactory/poor & & & & & 0.019 & 0.387 & 0.033 \\
\hline Counselling group & 40 & $195.3(24.9)$ & $165.4(73.3)$ & $202.3(24.5)$ & & & \\
\hline Health information group & 35 & $207.4(26.5)$ & $194.4(41.6)$ & $204.1(23.6)$ & & & \\
\hline MMSE 30-28 & & & & & 0.194 & 0.140 & 0.155 \\
\hline Counselling group & 61 & $207.5(26.3)$ & $188.4(56.3)$ & $201.1(24.9)$ & & & \\
\hline Health information group & 71 & $211.5(25.4)$ & $199.2(42.5)$ & $209.1(25.3)$ & & & \\
\hline MMSE 27-25 & & & & & 0.603 & 0.711 & 0.214 \\
\hline Counselling group & 40 & $199.0(25.8)$ & $183.0(57.2)$ & $202.8(23.9)$ & & & \\
\hline Health information group & 32 & $200.0(23.5)$ & $178.6(61.8)$ & $200.5(20.1)$ & & & \\
\hline SPPB 12-11 & & & & & 0.282 & 0.065 & 0.038 \\
\hline Counselling group & 64 & $211.5(23.8)$ & $199.3(44.0)$ & $213.0(23.2)$ & & & \\
\hline Health information group & 73 & $212.5(24.6)$ & $196.8(27.9)$ & $209.2(25.5)$ & & & \\
\hline SPPB $10-5$ & & & & & 0.313 & 0.919 & 0.464 \\
\hline Counselling group & 37 & $191.3(25.9)$ & $163.8(68.1)$ & $197.1(24.0)$ & & & \\
\hline Health information group & 30 & $196.7(23.5)$ & $183.1(54.3)$ & $200.7(19.5)$ & & & \\
\hline
\end{tabular}

Generalized estimation equations with $p$-values for the main effects of group and time, and group by time interaction are shown

other people and incorporated new knowledge on behavior change techniques into the intervention [12]. Motivation is a psychological concept defined as 'a driving force for the goal-directed behavior' [13]. Consequently, in the current study we aimed to promote autonomous motivation for selfselected activities [12]. Autonomous motivation relates to personal importance rather than extrinsic control [13].

The results showed that the secondary outcome 'will to act' did not change, but 'activity' increased. Activity was indicated as the sum of the accumulated participation in the 17 active behaviors forming the UJACAS activity subscore. Our expectation was that the counselling intervention component would mainly increase the subscore 'will to act' and that the booklet and newsletters would contribute to the changes of 'ability to act' and 'opportunity to act' respectively. As a result of the interaction of those elevated subscores the activity subscore would increase and the total score would increase. The results showed that even though the change of the secondary outcome 'will to act' was insignificant, the change of the 'activity' score was significant. The results may imply that even a small change in motivation may produce significant behavioral changes. However, this topic needs further research. The subscore 'opportunity to act' did not change. The 'ability to act' scores did not change either. The responses concerning ability to act clustered toward the higher values, suggesting, first, that there was not much room for change, and second, that the participants were well able to do the activities and thus inability to act did not prevent them from doing what they want to do. 
The preplanned subgroup analyses suggested that people with good lower extremity performance (SPPB score 11-12) or lower self-perceived financial situation benefitted more. However, all the observed effects were modest. These results should not be interpreted as intervention effects but rather they lay foundations for planning future studies.

There may be some explanations for the lower than expected intervention effect in the active aging score. It is possible that our inclusion criteria was not optimal. Our goal was to target the 'middle group' in terms of activity in order to find a group of people who had room for improvement and in the same time also resources to increase their activity. However, using the life-space mobility score between 52.3 and 90.0 on the University of Alabama at Birmingham Life-Space Assessment (LSA; [27, 28]) may have resulted in including people with highly active approach to life to start with. The subscale analyses suggested that the change in the total score resulted from the changes in the activity subscale. It is possible that the subscales relating to ability and opportunity to do different activities may not respond to a counselling intervention, but instead may require environmental modifications, e.g. changes in the physical living environment or social context. In the future in similar interventions, it might be better to choose the UJACAS activity subscore as the main outcome and the other subscores as secondary outcomes, because they may describe factors underlying activity. We recruited participants from a population-based probability sample, and the participants were not self-referred. This reduces bias towards higher intervention effectiveness often observed in studies among convenience samples. It is also possible that our active aging scale in terms of items and categorizations did not capture the changes caused by the intervention. As the items did not have strict external criteria for specific activities, it is possible that the activities changed to more demanding forms, but the scale did not detect that. It is also possible that the generic scale with 17 different activities made it difficult to capture changes, because each participant selected one to three activities that they aimed to increase. The anecdotal information from the participant feedback forms suggests that many participants perceived that the counselling promoted their active agency, some participants did not perceive a need for significant changes in their behavior, while some had so problematic issues in their lives that could not be solved by the current intervention. Finally, we need to admit that behavior change is difficult. A meta-analysis on the effectiveness of face-to-face interventions for promoting physical activity for at least at 12 months concluded that the effectiveness of these interventions was not supported by high quality studies [43].

The strengths of the current study include the randomized controlled design and incorporating into the intervention the up-to-date behavioral change techniques, such as setting goals and planning, getting feedback and monitoring, social support and monitoring consequences. Our study attrition was low. The main outcome was the novel active aging score not previously studied. The intervention lasted for one year. Consequently, the baseline and the final assessment took place in the same season. Even though the intervention effect was low, it is still notable that in our rather homogenous sample, there was a significant change considering that it is very difficult to change behaviors.

The weaknesses include that we do not yet know how big a change in the active aging score is meaningful. However, the responses are self-assessments, and any change in selfreport may be considered meaningful, because it is based on the participants' immediate experience about their life. We do not know whether the participants excluded from the study based on their too low or high life-space mobility score would have benefitted more from the intervention. The preplanned subgroup analyses suggest that the active aging score increased more among those with intact lower extremity performance and lower self-rated financial situation. Excluding people with lower MMSE scores might not have been necessary, because the subgroup analyses showed that the results did not differ between those in the higher or lower MMSE score.

An individualized counselling intervention based on self-determination theory and theory of planned behavior, and consisting of one face-to-face session, an information booklet, four phone counselling sessions and four printed newsletters featuring the available activities organized in our town increased the active aging score modestly compared to the health information intervention. These findings suggest that it is possible to promote active aging by focusing on the individuals' goals. Future studies on active aging should include samples with higher variability and incorporate environmental interventions to influence opportunities in addition to individual-based intervention.

Acknowledgements Open access funding provided by University of Jyväskylä (JYU). The AGNES study was financially supported by an Advanced Grant from the European Research Council (Grant 693045 to Taina Rantanen) and the Academy of Finland (Grant 310526 to Taina Rantanen). The funders had no role in the design of the study and data collection, analysis and interpretation of data, and in writing the manuscript. The content of this article does not reflect the official opinion of the European Union. Responsibility for the information and views expressed in the article lie entirely with the author(s).

Data availability After completion of the study, data will be stored at the Finnish Social Science Data Archive without potential identifiers (open access). Until then, pseudonymized datasets are available to external collaborators upon agreement on the terms of data use and publication of results. To request the data please contact Professor Taina Rantanen (taina.rantanen@jyu.fi).

\section{Compliance with ethical standards}

Conflict of interest The authors declare no conflict of interest. 
Ethical approval All procedures performed were in accordance with the ethical standards of the 1964 Helsinki declaration and its later amendments. The ethical committee of the Central Finland Hospital district provided an ethical statement pertaining to the AGNES cohort and intervention studies on August 23, 2017.

Informed consent All participants signed an informed consent prior to the start of the study.

Open Access This article is licensed under a Creative Commons Attribution 4.0 International License, which permits use, sharing, adaptation, distribution and reproduction in any medium or format, as long as you give appropriate credit to the original author(s) and the source, provide a link to the Creative Commons licence, and indicate if changes were made. The images or other third party material in this article are included in the article's Creative Commons licence, unless indicated otherwise in a credit line to the material. If material is not included in the article's Creative Commons licence and your intended use is not permitted by statutory regulation or exceeds the permitted use, you will need to obtain permission directly from the copyright holder. To view a copy of this licence, visit http://creativecommons.org/licenses/by/4.0/.

\section{References}

1. Halaweh H, Dahlin-Ivanoff S, Svantesson U et al (2018) Perspectives of older adults on aging well: a focus group study. J Aging Res. https://doi.org/10.1155/2018/9858252

2. Guell C, Shefer G, Griffin S et al (2016) 'Keeping your body and mind active': an ethnographic study of aspirations for healthy aging. BMJ Open 6:e009973. https://doi.org/10.1136/bmjop en-2015-009973

3. Saajanaho M, Rantakokko M, Portegijs E et al (2016) Life resources and personal goals in old age. Eur J Aging 1:195-208. https://doi.org/10.1007/s10433-016-0382-3

4. Duppen D, Lambotte D, Dury S et al (2019) Social participation in the daily lives of frail older adults: types of participation and influencing factors. J Gerontol B. https://doi.org/10.1093/geronb/ gbz045

5. UNECE statistics wikis (2017) Active aging index. https://stats wiki.unece.org/display/AAI/Active+Aging +Index +Home. Accessed 24 Apr 2018

6. WHO (2002) Active aging. A policy framework. World Health Organization, Geneva

7. Rantanen T, Portegijs E, Kokko K et al (2019) Developing an assessment method of active aging: University of Jyvaskyla active aging scale. J Aging Health. https://doi.org/10.1177/0898264317 750449

8. International Classification of Functioning, Disability and Health. World Health Organization. Geneva 2001. https://apps.who.int/ iris/bitstream/handle/10665/42407/9241545429.pdf. Accessed 15 Aug 2017

9. Skevington SM, Lotfy M, O'Connell KA (2004) The World Health Organization's WHOQOL-BREF quality of life assessment: psychometric properties and results of the international field trial. A report from the WHOQOL group. Qual Life Res 13:299-310. https://doi.org/10.1023/B:QURE.0000018486.91360 .00

10. Van Malderen L, Mets T, Gorus E (2012) Interventions to enhance the quality of life of older people in residential long-term care: a systematic review. Aging Res Rev 12:141-150

11. Rantanen T, Äyräväinen I, Eronen J et al (2015) The effect of an outdoor activities' intervention delivered by older volunteers on the quality of life of older people with severe mobility limitations: a randomized controlled trial. Aging Clin Exp Res 27:161-169. https://doi.org/10.1007/s40520-014-0254-7

12. Rantanen T, Pynnönen K, Saajanaho M et al (2019) Individualized counselling for active aging: protocol of a single-blinded, randomized controlled trial among older people (the AGNES intervention study). BMC Geriatr. https://doi.org/10.1186/s1287 7-018-1012-z

13. Ryan RM, Deci EL (2000) Self-determination theory and the facilitation of intrinsic motivation, social development, and wellbeing. Am Psychol 55:68-78

14. Ajzen I (1991) The theory of planned behavior. Organ Behav Hum Decis Process 50:179-211

15. Vallerand RJ, O'Connor BP, Hamel M (1995) Motivation in later life: theory and assessment. Int J Aging Hum Dev 41:221-238

16. Vallerand RJ, O'Connor BP (1989) Motivation in the elderly: a theoretical framework and some promising findings. Can Psychol 30:538

17. Steverink N, Lindenberg S, Slaets JPJ (2005) How to understand and improve older people's self-management of wellbeing. Eur J Aging 2:235

18. White KM, Terry DJ, Troup C et al (2012) An extended theory of planned behavior intervention for older adults with type 2 diabetes and cardiovascular disease. J Aging Phys Act 20:281

19. Ahmad MH, Shahar S, Teng NIMF et al (2014) Applying theory of planned behavior to predict exercise maintenance in sarcopenic elderly. Clin Interv Aging 9:1551-1561

20. Sjoberg S, Kim K, Reicks M (2004) Applying the theory of planned behavior to fruit and vegetable consumption by older adults. J Nutr Elder 23:35-46

21. Gretebeck KA, Black DR, Blue CL et al (2007) Physical activity and function in older adults: theory of planned behavior. Am J Health Behav 31:203-2014

22. Stolte E, Hopman-Rock M, Aartsen MJ et al (2017) The theory of planned behavior and physical activity change: outcomes of the aging well and healthily intervention program for older adults. J Aging Phys Act 25:438-445

23. Hagger MS, Chatzisarantis NLD (2009) Integrating the theory of planned behaviour and self-determination theory in health behaviour: a meta-analysis. Br J Health Psychol 14:275-302

24. Chung P-K, Zhang C-Q, Liu J-D et al (2017) The process by which perceived autonomy support predicts motivation, intention, and behavior for seasonal influenza prevention in Hong Kong older adults. BMC Public Health 18:1-9

25. Rantanen T, Saajanaho M, Karavirta L et al (2018) Active aging-resilience and external support as modifiers of the disablement outcome: AGNES cohort study protocol. BMC Public Health 18:565. https://doi.org/10.1186/s12889-018-5487-5

26. Portegijs E, Karavirta L, Saajanaho M et al (2019) Assessing physical performance and physical activity in large population-based aging studies: home-based assessments or visits to the research center? BMC Public Health 19:1570. https://doi. org/10.1186/s 12889-019-7869-8

27. Baker PS, Bodner EV, Allman RM (2003) Measuring life-space mobility in community-dwelling older adults. J Am Geriatr Soc 51:1610-1614

28. Portegijs E, Rantakokko M, Viljanen A et al (2016) Identification of older people at risk of ADL disability using the lifespace assessment: a longitudinal cohort study. J Am Med Dir Assoc 17:410-414

29. Folstein MF, Folstein SE, McHugh PR (1975) "Mini-mental state": a practical method for grading the cognitive state of patients for the clinician. J Psychiatr Res 12:189-198

30. Craig P, Dieppe P, Macintyre S et al (2013) Developing and evaluating complex interventions: the new medical research council guidance. Int J Nurs Stud 50:587-592 
31. Eccles MP, Grimshaw JM, MacLennan G et al (2012) Explaining clinical behaviors using multiple theoretical models. Implement Sci 7:99. https://doi.org/10.1186/1748-5908-7-99

32. French SD, Green SE, O'Connor DA et al (2012) Developing theory-informed behaviour change interventions to implement evidence into practice: a systematic approach using the theoretical domains framework. Implement Sci 7:38. https://doi. org/10.1186/1748-5908-7-38

33. Michie S, van Stralen MM, West R (2011) The behaviour change wheel: a new method for characterising and designing behaviour change interventions. Implement Sci 6:42. https://doi. org/10.1186/1748-5908-6-42

34. Bowling A, Hankins M, Windle G et al (2013) A short measure of quality of life in older age: the performance of the brief older people's quality of life questionnaire (OPQOL-brief). Arch Gerontol Geriatr 56:181-187

35. Finland S (2007) Education in Finland: more education for more people. The statistics Finland publication in series "Finland 19172007. The Finnish independence in statistics"

36. Rantanen T, Portegijs E, Viljanen A et al (2012) Individual and environmental factors underlying life space of older peoplestudy protocol and design of a cohort study on life-space mobility in old age (LISPE). BMC Public Health 12:1018

37. Guralnik JM, Simonsick EM, Ferrucci L et al (1994) A short physical performance battery assessing lower extremity function: association with self-reported disability and prediction of mortality and nursing home admission. J Gerontol 49:M85-94

38. Saajanaho M, Rantakokko M, Portegijs E et al (2015) Personal goals and changes in life-space mobility among older people. Prev Med 81:163-167
39. Pynnönen K, Törmäkangas T, Rantanen T et al (2018) Effect of a social intervention of choice vs. control on depressive symptoms, melancholy, feeling of loneliness, and perceived togetherness in older Finnish people: a randomized controlled trial. Aging Ment Health 22:77-78

40. Portegijs E, Rantakokko M, Mikkola TM et al (2014) Association between physical performance and sense of autonomy in outdoor activities and life-space mobility in community-dwelling older people. J Am Geriatr Soc 62:615-621

41. Leinonen R, Heikkinen E, Hirvensalo M et al (2007) Customeroriented counseling for physical activity in older people: study protocol and selected baseline results of a randomized-controlled trial (ISRCTN 07330512). Scand J Med Sci Sports 17:156-164

42. Rasinaho M, Hirvensalo M, Törmäkangas T et al (2012) Effect of physical activity counseling on physical activity of older people in Finland (ISRCTN 07330512). Health Promot Int 27:463-474

43. Richards J, Hillsdon M, Thorogood M et al (2013) Face-to-face interventions for promoting physical activity. Cochrane Database Syst Rev. https://doi.org/10.1002/14651858.CD010392.pub2/full

Publisher's Note Springer Nature remains neutral with regard to jurisdictional claims in published maps and institutional affiliations. 